\title{
Identifying birth places of high-velocity stars
}

\author{
V. Hambaryan ${ }^{1,2} *$, R. Neuhäuser ${ }^{1}$ \\ ${ }^{1}$ Astrophysical Institut and University Observatory Jena, Germany \\ ${ }^{2}$ NAS RA V. Ambartsumian Byurakan Astrophysical Observatory (BAO), Armenia \\ ${ }^{*}$ E-mail: Valeri.Hambaryan@uni-jena.de
}

\begin{abstract}
We searched for the high-velocity and isolated neutron stars that encountered in the past with a stellar cluster/association closer than 20 pc. We took about 830000 stars with the high-quality astrometry and radial velocities from the Gaia DR2 catalogue and empirically selected about 560 high-velocity stars.

We used a full gravitational potential of the Galaxy to calculate the motion of a stellar cluster/association and a candidate of high-velocity star from their current positions to the proximity epoch. For this calculations we used a numerical integration in rectangular, Galactocentric coordinates. We used a covariance matrices of the astrometric data for each star to estimate the accuracy of the obtained proximity distance and epoch. For this aim we used a Monte Carlo method, replaced each star with 10000 of its simulations and studied the distribution of their individual close passages near a stellar cluster/association. In addition we investigated a neutron star/runaway star pairs very likely both ejected from binary system during supernova event.
\end{abstract}

Keywords: high-velocity stars - runaway stars - hyper-velocity stars - neutron stars - stellar associations - stellar clusters - Gaia astrometry. 


\section{Introduction}

What are high-velocity stars? Depending on the definition, a highvelocity star is a star moving faster than $60-100 \mathrm{~km} / \mathrm{s}$ relative to the average motion of the stars in its $\left(\mathrm{V}_{\text {Sun }}=18.0-20.0 \mathrm{~km} / \mathrm{s}\right)$ neighbourhood. Obviously, this definition is somewhat arbitrary and it depends on the many factors (for example, star mass, age, origin, galactic potential, velocity distribution model, etc.). Therefore, empirical determination of them might be more robust given the huge number of stars with reliable astrometric parameters and radial velocities provided by Gaia mission.

In general, there are three subclasses for high-velocity stars and they have different origin.

- Runaway stars: First of all, so called, "Runaway stars" are subclass of high velocity stars and were first introduced as $\mathrm{O}$ and $\mathrm{B}$ type stars by Blaauw [5]. Runaway stars are thought to have formed in the stellar associations[2] and ejected into the general Galactic field. The proper motion of a runaway star often points exactly away from a stellar association, of which the star was formerly a member. The majority of runaway stars in the literature are high-mass $\mathrm{O}$ and $\mathrm{B}$ type stars with ejection velocities less than $200 \mathrm{kms}^{-1}$ [23]. Recent results show it is possible for low-mass $\mathrm{G} / \mathrm{K}$ type stars with ejection velocities up to $\sim 1300 \mathrm{kms}^{-1}[26]$.

- Hypervelocity stars (HVSs): are another subclass of high velocity stars, the fastest stars in our Galaxy, which have extreme velocities above the escape speed of the Milky Way. HVSs can obtain their large velocity from a number of different processes. Hills [16] first theoretically predicted the formation of HVSs via three-body interactions between a binary star system and the massive nucleous in the Galactic Center (GC). Hypervelocity stars are stars with velocities that are substantially different from that expected for a star belonging to the normal distribution of stars in a galaxy. Such stars may have velocities on the order of $1000 \mathrm{~km} / \mathrm{s}$.

- Pulsars: Some neutron stars are inferred to be traveling with similar speeds as HVSs. This could be related to runaway-stars and their ejection mechanism. Neutron stars are the remnants of supernova explosions, and their extreme speeds are very likely the result of an asymmetric supernova explosion or the loss of their near partner during the supernova explosions that forms them. 


\section{Origin mechanisms}

The Binary Ejection Mechanism (BEM): this was first proposed by Blaauw (1961) to explain the ejection of runaway O and B stars out of galactic plane. In this scenario the secondary star of a close binary receives its ejection velocity when the primary explodes as a supernova.

The Dynamical Ejection Mechanism (DEM): this was first proposed by Ambartsumian [1] in a Trapezium type young multiple systems and by Poveda, Ruiz \& Allen [24] as an alternative to BEM to produce runaway stars. Dynamical interactions between stars inside young, open clusters and associations can give large kicks to one or both stars in a collision.

\section{Selection of candidates of high-velocity stars}

The recent release of the Gaia DR2 catalog [9] has renewed interest in the high-velocity stars in the Galaxy. In addition to contributing new samples of candidates [e.g., 19, 25, 12, 14], Gaia proper motions and radial velocities allow more robust assessments of the distances and space velocities of previously identified high velocity stars.

The positions and velocities available for over 7 million sources in the Gaia DR2 archive also enable new tests of theoretical models for the highest velocity stars.

Here, we use the Gaia DR2 proper motion and radial velocity data for the brightest and nearby (up to $3 \mathrm{kpc}$ from the Sun) stars to select highvelocity candidates and identify possible birth place and counterparts.

Identifying true high-velocity outliers in the Gaia DR2 archive is challenging. Among the roughly 7 million stars with measured parallax, proper motion, and radial velocity, no more than a few hundred candidates emerge with Galactic rest-frame velocity close to or exceeding the local escape velocity [e.g., 20, 12]. Based on the quoted errors of the measured quantities, first we have followed the recommendations in the basic source parameter descriptions $[9,7,17]$ by requiring for high-quality astrometry and quality of the reported radial velocity.

Next, we have selected Gaia DR2 sources for which astrometric parameters (positions, parallax and proper motions) and radial velocity have relative errors not exceeding $10 \%$. Further, these sources converted into Galacto-centric cartesian coordinates $(\mathrm{X}, \mathrm{Y}, \mathrm{Z}, \mathrm{U}, \mathrm{V}, \mathrm{W})$ and analysed empirical distribution of total velocities in a spherical rings of $5 \mathrm{pc}$ width around center of the Galaxy. The estimates of distribution parameters (the median, most probable value, the highest posterior density intervals ${ }^{1}$ ) of total

\footnotetext{
${ }^{1}$ The highest posterior density interval (HPD) determined as a probabilistic region
} 


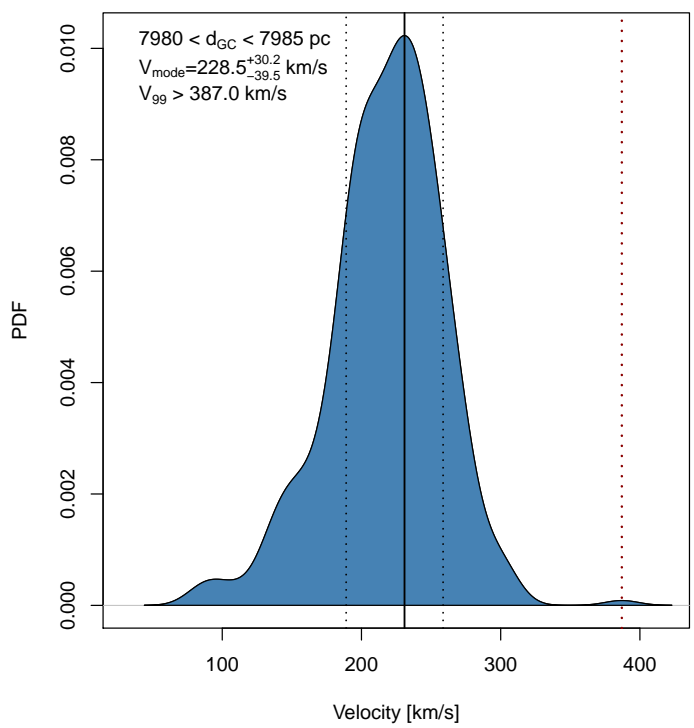

Figure 1: Selection procedure of highvelocity candidates in the spherical ring of 7980 $<d_{G C}<7985 \mathrm{pc}$ around center of the Galaxy. By vertical dashed (darkred, $\left.V_{99}=387.0 \mathrm{~km} / \mathrm{s}\right)$ line indicated the selection criteria of high-velocity candidate stars

velocity in a spherical ring, having at least 100 sources, performed with kernel-density estimates obtained with the function density in $\mathrm{R}$ (an example is illustrated in Figure 1). From this distribution as a high-velocity candidates were selected outliers which have a value $V_{\text {total }}>V_{H P D_{h i}}(99 \%)$ (c.f., vertical darkred-dashed line in the Figure 1 for the spherical ring at the distance of $7980<d_{G C}<7985$ pc from GC, containing 2955 stars, $68 \%$ of which in the interval of [189.0 - 258.7] km/s and only 6 stars exceeding $\left.V_{H P D_{h i}}(99 \%)=387.0 \mathrm{~km} / \mathrm{s}\right)$. Note, that this is a conservative criteria with higher confidence for the selection of a high-velocity candidate star and by an application of a different methods (e.g., the Mahalanobis distance distance or $3-\sigma$ clipping alogorithm) lead to the similar results. With above-mentioned procedure, we have selected 557 high-velocity (see, Fig. 2) candidate stars (367 within $3 \mathrm{kpc}$ distance from the Sun) not included also in the list of so-called potentially contaminated radial velocity stars [6].

\section{Motion of stars in the Galaxy}

To study the Galactocentric motion of a high-velocity star we use a numerical integration of its equations of motion (Eq. 1) in the gravitational field of the Galaxy:

around a posterior mode, and is similar to a confidence interval in a classical statistics [see e.g. 11]. 

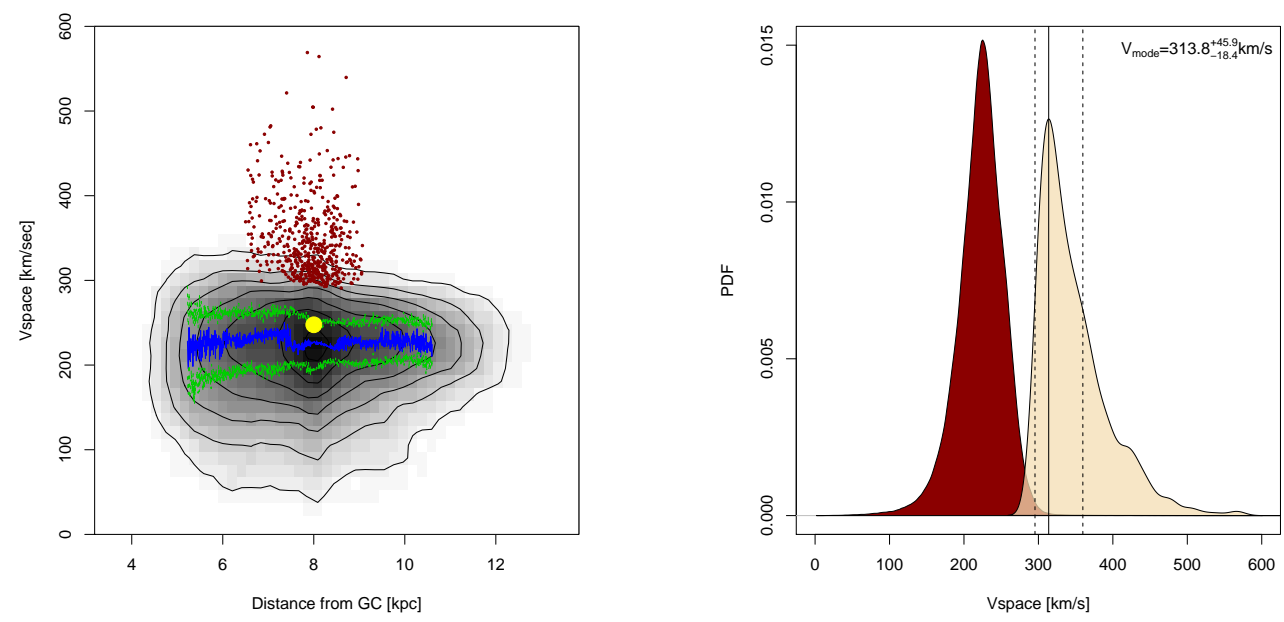

Figure 2: Selected high-velocity candidate stars based on the empirical distribution function of space velocities of Gaia DR2 catalog. Left panel : Total velocity in the Galactic rest-frame as a function of Galactocentric distance for all selected the 828098 stars in Gaia DR2 with high quality astrometric and 10- $\sigma$ data (see details in the text). Grey colour level is proportional to the logarithmic number density of stars. The blue and green solid lines are the most probable value and $\operatorname{HPD}(68 \%)$ interval of total velocity of stars derived from empirical probability distribution function using kernel-density estimator. The dark red points are selected candidates of 557 high-velocity stars also derived from the same probability density function with $\operatorname{Prob}\left(V_{\text {total }}\right)>99 \%$. The yellow filled dot presents the Sun position in the diagram. Right panel: A normalized probability density function of distributions of velocities of all (dark red) and selected as highvelocity candidate stars. 


$$
\begin{aligned}
\ddot{x} & =-\frac{\partial}{\partial x} \Phi(x, y, z), \\
\ddot{y} & =-\frac{\partial}{\partial y} \Phi(x, y, z), \\
\ddot{z} & =-\frac{\partial}{\partial z} \Phi(x, y, z),
\end{aligned}
$$

where gravitational potential $\Phi(x, y, z)$ is expressed in rectangular coordinates. Here, we considered widely used the Milky-Way gravitational potential model. This model of gravitational potential $\Phi(x, y, z)$ is the sum of a central bulge component $\Phi_{b}(x, y, z)$ (spherically symmetric), an axisymmetric disk $\Phi_{d}(x, y, z)$ and a massive spherical Galactic halo $\Phi_{h}(x, y, z)$ (dark matter included):

$$
\Phi(x, y, z)=\Phi_{b}(x, y, z)+\Phi_{d}(x, y, z)+\Phi_{h}(x, y, z),
$$

where $(x, y, z)$ are Galactocentric cartesian coordinates.

For the Galactic bulge and disk components we use formulae $\left(\Phi_{b}(r)\right.$, Hernquist bulge, [15] and $\Phi_{d}(R, z)$, Miyamoto-Nagai disk, [21]):

$$
\begin{gathered}
\Phi_{b}(r)=-\frac{G M_{b}}{\sqrt{r^{2}+b_{b}^{2}}}, \\
\Phi_{d}(R, z)=-\frac{G M_{d}}{\sqrt{R^{2}+\left(a_{d}+\sqrt{z^{2}+b_{d}^{2}}\right)^{2}}},
\end{gathered}
$$

and for a massive spherical Galactic halo $\left(\Phi_{h}(r)\right.$, Navarro-Frenk-White halo, $[22])$ :

$$
\Phi_{h}(r)=-\frac{G M_{h}}{r} \ln \left(1+\frac{r}{a_{h}}\right),
$$

where $r^{2}=x^{2}+y^{2}+z^{2}$ and $R^{2}=x^{2}+y^{2}$, with parameters of $M_{b}=$ $(0.103 \pm 0.006) \times 10^{11} M_{\odot}, M_{d}=(0.650 \pm 0.020) \times 10^{11} M_{\odot}, M_{h}=(2.900 \pm$ $0.765) \times 10^{11} M_{\odot}, b_{b}=(0.267 \pm 0.009), a_{d}=(4.40 \pm 0.73), b_{d}=(0.308 \pm 0.005)$, and $a_{h}=(7.7 \pm 2.1)$ in kpc (Model III from Bajkova \& Bobylev [4]), derived by fitting of modern data on circular velocities of Galactic objects located at distances up to $200 \mathrm{kpc}$ from the Galactic center.

For numerical integration we utilise the fast and accurate Gauss-Everhart orbit integrator provided by Avdyushev [3].

\subsection{Simulation of astrometric parameters}

In order to estimate the uncertainty of the close stellar passage parameters we replace each considered star with a large number (typically 10000 ) of its simulations, drawn from a multivariate normal distribution. This is 
possible because in Gaia DR2 catalogue included full information on the covariance matrix of astrometric parameters. Such a procedure possesses an evident superiority over the individual, independent random drawing of each parameter what ignores their obvious mutual dependence. To generate random vectors $\mathbf{x}$, i.e. of proper motions, parallax, and radial velocity, from a multivariate normal distribution $\mathcal{N}_{n}(\mathbf{m}, \Sigma)$ with a given vector of means $\mathbf{m}$ and a given covariance matrix $\boldsymbol{\Sigma}$, we used mvtnorm : a Multivariate Normal and t Distributions R package with Cholesky method, developed by Genz et al. [10]:

$$
\begin{gathered}
\mathbf{m}=\left[\mu_{\alpha *}, \mu_{\delta}, \varpi, v_{\mathrm{rad}}\right] \\
\Sigma=\left(\begin{array}{cccc}
\sigma_{\mu_{\alpha *}}^{2} & \sigma_{\mu_{\alpha *}} \sigma_{\mu_{\delta}} \rho\left(\mu_{\alpha *}, \mu_{\delta}\right) & \sigma_{\mu_{\alpha *}} \sigma_{\varpi} \rho\left(\mu_{\alpha *}, \varpi\right) & 0.0 \\
\sigma_{\mu_{\alpha *}} \sigma_{\mu_{\delta}} \rho\left(\mu_{\alpha *}, \mu_{\delta}\right) & \sigma_{\mu_{\delta}}^{2} & \sigma_{\mu_{\delta}} \sigma_{\varpi} \rho\left(\mu_{\delta}, \varpi\right) & 0.0 \\
\sigma_{\mu_{\alpha *}} \sigma_{\varpi} \rho\left(\mu_{\alpha *}, \varpi\right) & \sigma_{\mu_{\delta}} \sigma_{\varpi} \rho\left(\mu_{\delta}, \mu_{\varpi}\right) & \sigma_{\varpi}^{2} & 0.0 \\
0.0 & 0.0 & 0.0 & \sigma_{v_{\mathrm{rad}}}^{2}
\end{array}\right),
\end{gathered}
$$

where $\rho$ denotes the correlation coefficient between the astrometric parameters, and it is provided in the Gaia DR2 catalogue. Radial velocities are uncorrelated to the astrometric parameters, and we assume them to follow a Gaussian distribution centered on $v_{\text {rad }}$, and with standard deviation $\sigma_{v_{\text {rad }}}$.

In order to determine most likely backward in time stellar trajectories of candidates of a high-velocity stars and their potential birth places (stellar cluster/association or hypothetical supernova), we used as a measure a multivariate Gaussian likelihood in four dimensional space (time and position):

$$
L(\mu, \Sigma \mid Z)=\prod_{i=1}^{n} L_{i}\left(\mu_{i}, \Sigma_{i} \mid z_{i}\right)=\prod_{i=1}^{n}(2 \pi)^{-\frac{1}{2} p_{i}}\left|\Sigma_{i}\right|^{-\frac{1}{2}} e^{\left(z_{i}-\mu_{i}\right) \Sigma_{i}^{-1}\left(z_{i}-\mu_{i}\right)^{T}}
$$

\subsection{Runaway stars AE aur, $\mu \mathrm{Col}$ and 53 Eri and star forming region Orion}

AE Aur, $\mu$ Col, and 53 Ari are runaway stars traced to the Trapezium cluster in the Orion Nebula two million years ago. These stars might have been ejected during a collision of two binary star groups Hoogerwerf et al. [13]. For the testing purpose, we have applied our approach to these stars and young clusters in Orion star forming region (see Table 1,2 and Figure 3).

\section{Preliminary results}

In order to identify the most probable parent stellar association/cluster of a high-velocity candidate stars we used updated catalogue of optically 


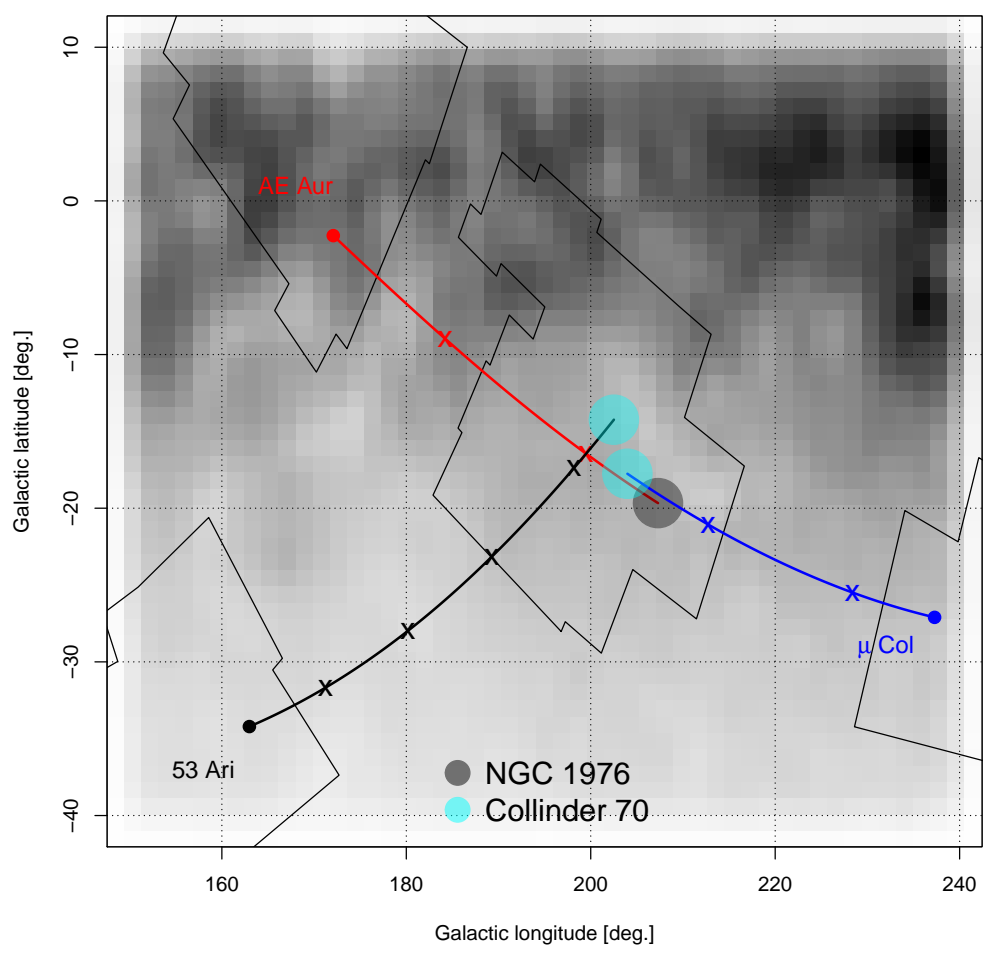

Figure 3: Simulated closest approach of stellar trajectories of runaway stars AE Aur, $\mu$ Col, 53 Ari and young stellar groups (NGC 1976, 1977, 1980, 1981 and Collinder 70) in Orion complex star forming region. The most probable traceback stellar trajectories, birth places and ejection times (highest likelihoods) of these runaway stars are presented in the bottom right panel (see also Table 2). 


\begin{tabular}{lccccccc}
\hline Name & $\begin{array}{c}\text { RA } \\
{\left[{ }^{\circ}\right]}\end{array}$ & $\begin{array}{c}\text { DEC } \\
{\left[{ }^{\circ}\right]}\end{array}$ & $\begin{array}{c}\mu_{\alpha} \cos \delta \\
{[\mathrm{mas} / \mathrm{yr}]}\end{array}$ & $\begin{array}{c}\mu_{\delta} \\
{[\mathrm{mas} / \mathrm{yr}]}\end{array}$ & $\begin{array}{c}\text { RV } \\
{[\mathrm{km} / \mathrm{s}]}\end{array}$ & $\begin{array}{c}\mathrm{d} \\
{[\mathrm{pc}]}\end{array}$ & $\begin{array}{c}\lg (\text { Age }) \\
{[\text { year }]}\end{array}$ \\
\hline NGC 1976 & 83.82 & -5.39 & 1.96 & -0.77 & 27.80 & 400.0 & 7.71 \\
NGC 1977 & 83.85 & -4.82 & 2.42 & -1.90 & 24.20 & 500.0 & 7.08 \\
NGC 1980 & 83.85 & -5.91 & 0.83 & -0.36 & 23.00 & 550.0 & 6.67 \\
NGC 1981 & 83.79 & -4.40 & 1.01 & 1.14 & 27.90 & 400.0 & 7.50 \\
Collinder 70 & 83.88 & -1.10 & 0.36 & -0.68 & 31.70 & 390.0 & 6.71 \\
\hline HIP24575(AE Aur) & 79.076 & 34.313 & -4.440 & 43.368 & 56.7 & 405.81 & \\
HIP27204( $\mu$ Col) & 86.500 & -32.307 & 2.988 & -22.030 & 109.0 & 465.64 & \\
HIP14514(53 Ari) & 46.857 & 17.880 & -23.268 & 7.973 & 21.7 & 315.77 & \\
\hline
\end{tabular}

Table 1: Input parameters for simulation of young clusters in Orion star forming region and runaway stars AE Aur, $\mu$ Col, and 53 Ari.

\begin{tabular}{lrrcc}
\hline $\begin{array}{l}\text { Cluster } \\
\text { name }\end{array}$ & $\begin{array}{r}\text { Runaway } \\
\text { name }\end{array}$ & $\begin{array}{r}\text { Relative } \\
\text { number }[\%]\end{array}$ & $\begin{array}{c}\text { Most probable } \\
\text { time }[\mathrm{Myr}]\end{array}$ & $\begin{array}{c}\text { Time range } \\
{[\mathrm{Myr}]}\end{array}$ \\
\hline NGC 1976 & AE Aur(HIP24575) & 22.30 & -2.48 & $-2.66-2.26$ \\
NGC 1981 & AE Aur(HIP24575) & 16.70 & -2.52 & $-2.71-2.31$ \\
Collinder 70 & AE Aur(HIP24575) & 10.70 & -2.25 & $-2.38-2.12$ \\
Collinder 70 & $\mu$ Col(HIP27204) & 4.26 & -2.46 & $-2.69-2.23$ \\
Collinder 70 & 53 Ari(HIP14514) & 2.36 & -4.59 & $-5.09-4.21$ \\
NGC 1981 & $\mu$ Col(HIP27204) & 1.30 & -2.29 & $-2.47-2.15$ \\
NGC 1976 & $\mu$ Col(HIP27204) & 0.93 & -2.36 & $-2.49-2.20$ \\
NGC 1977 & AE Aur(HIP24575) & 0.77 & -2.48 & $-2.57-2.35$ \\
\hline
\end{tabular}

Table 2: Results of simulation of young clusters in Orion star forming region and runaway stars $\mathrm{AE}$ Aur, $\mu \mathrm{Col}$, and 53 Ari.

visible open clusters and candidates [8] consisting of 710 objects with astrometric parameters and radial velocity of the stellar system and traced back in time up to 30 Myrs with the step of 1000 yrs.

The similar procedure was applied also for pulsars [18] having good astrometric parameters, i.e. the distance and proper motions have relative errors not exceeding $10 \%$. In this case radial velocity of a pulsar was simulated from a uniform distribution in the range of -1000 to $1000 \mathrm{~km} / \mathrm{s}$.

\section{Summary}

We searched for the high-velocity stars from the Gaia DR2 catalogue and selected about 560 candidates.

We developed a new software to trace back the motion of stars through the Galactic potential, tested it successfully with the original case runaway stars AE Aur, mu Col, and 53 Ari and then applied it to above mentioned 

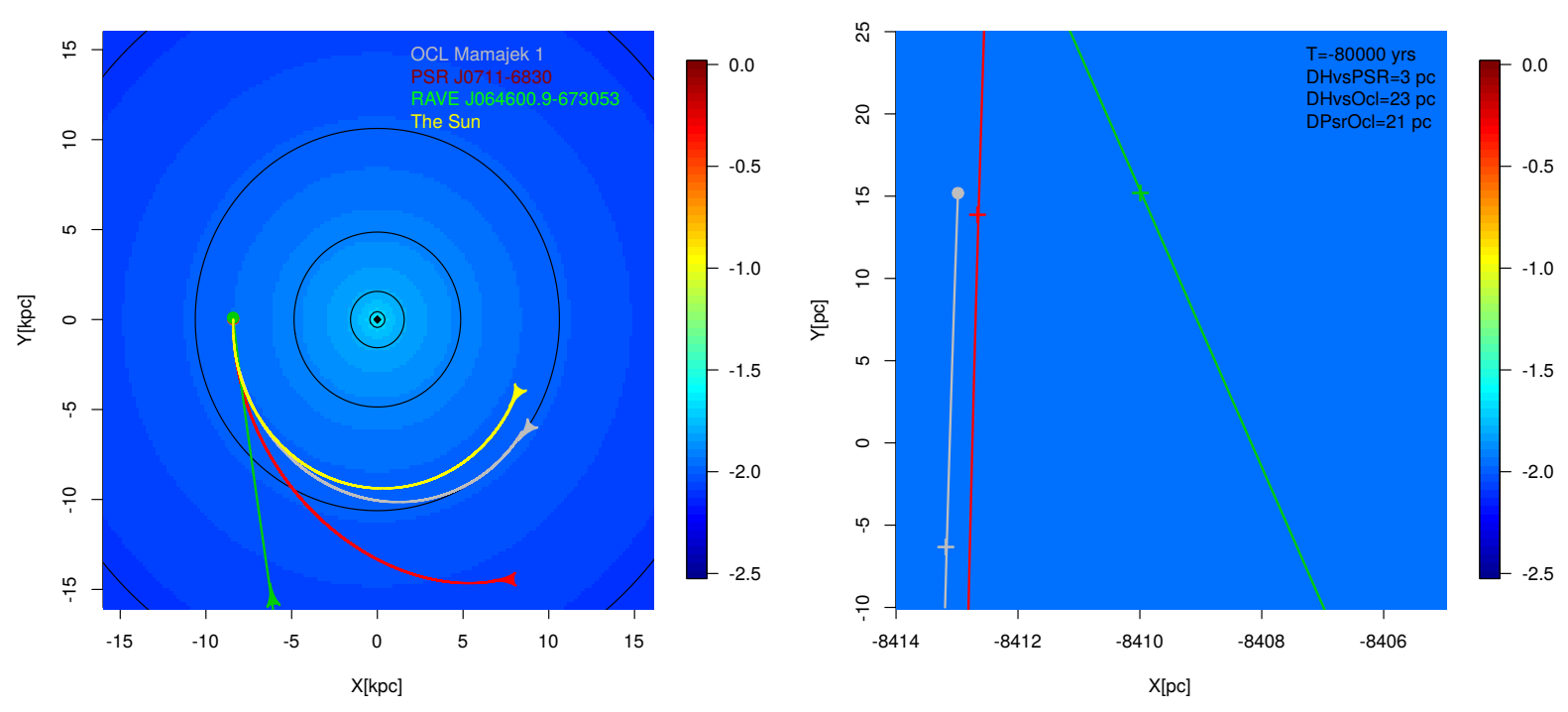

Figure 4: Possible triple associted with each other: star RAVE J064600.9673053=CD-67 470=Gaia2 5280169713520753536, OCL Mamajek 1 and PSR J0711-6830

high-velocity stars and optically visible open clusters.

It turned out that for $\sim 15 \%$ of high-velocity stars can be identified parent stellar cluster/association.

In some case we found also a plausible pulsar born in a possible supernova event (see Table 4 and Fig. 4).

\section{References}

[1] Ambartsumian, V. A. 1954, Communications of BAO, 1954, 15, 3.

[2] Ambartsumian, V. A. 1955, The Observatory, 75, 72.

[3] Avdyushev, V. A.Vychisl.Tekhnol.Journal Profile 15, No. 4, 31-46, 2010

[4] Bajkova and Bobylev, Open Astronomy, 26, 72, 2017, arXiv:1801.08875

[5] Blaauw, A. 1961, BAN, 15, 265

[6] Boubert, D., Strader, J., Aguado, D., et al. 2019, MNRAS, 280.

[7] Bromley, B.C., Kenyon, S.J., Brown, W.R., Geller, M. J. ,2018, 868, 25

[8] Dias, W. S., Alessi, B. S., Moitinho, A. \& Lépine, J. R. D., 2002, A\&A, 389,871 


\begin{tabular}{llrc}
\hline $\begin{array}{l}\text { Cluster } \\
\text { name }\end{array}$ & $\begin{array}{l}\text { Gaia DR2 } \\
\text { name }\end{array}$ & $\begin{array}{r}\mathrm{D}_{\min } \\
\text { number }[\mathrm{pc}]\end{array}$ & $\begin{array}{c}\text { Most probable } \\
\text { time }[\mathrm{Myr}]\end{array}$ \\
\hline FSR0237 & 26381685304316212480 & 0.22 & -4.22 \\
Dolidze52 & 25229899212967139200 & 0.67 & -6.10 \\
Trumpler & 2262053757371463808 & 0.87 & -0.75 \\
Turner 9 & 2940306024863622400 & 0.96 & -2.75 \\
Kronberger 13 & 2480690918702319104 & 1.30 & -5.12 \\
Dolidze 44 & 26544977963748689920 & 1.44 & -5.02 \\
Berkeley 87 & 25973898809770425344 & 1.73 & -2.35 \\
\hline
\end{tabular}

Table 3: Most probable parent open cluster of some high-velocity candidate stars.

\begin{tabular}{lllccc}
\hline Cluster & Gaia DR2 & Rave & PSR & $\mathrm{D}_{\min }(\mathrm{HP})$ & $\begin{array}{c}\text { Most probab } \\
\text { name [Myr] }\end{array}$ \\
name & name & name & & {$[\mathrm{pc}]$} & time \\
Mamajek 1 & 5280169713520753536 & J064600.9-673053 & J0711-6830 & -0.08 & 3.0 \\
Collinder 132 & 5605471258084062464 & J072858.5-301147 & J0630-2834 & -0.60 & 3.5 \\
\hline
\end{tabular}

Table 4: Most probable parent open cluster, high-velocity candidate stars abd pulsars.

[9] Gaia Collaboration, (Brown, A. G. A., et al.) 2018, A\&A, arXiv:1804.09365

[10] Alan Genz, Frank Bretz, Tetsuhisa Miwa, Xuefei Mi, Friedrich Leisch, Fabian Scheipl, Torsten Hothorn (2018). mvtnorm: Multivariate Normal and t Distributions. R package version 1.0-8.

[11] Gregory P. C., Loredo T. J., 1992, ApJ, 398, 146

[12] Hattori, K., Valluri, M., Bell, E. F., \& Roederer, I. U. 2018, ArXiv e-prints, arXiv:1805.03194

[13] Hoogerwerf, R.; De Bruijne, J. H. J.; De Zeeuw, P. T. 2001 A \& A, 365 49.

[14] Irrgang, A., Kreuzer, S., \& Heber, U. 2018, Astronomy and Astrophysics, 620, A48.

[15] Hernquist L., 1990, ApJ,356,359

[16] Hills, J.G., 1988, Nature, 331, 687

[17] Lindegren, L., Hernández, J., Bombrun, A., et al. 2018, AJ, 616, A2

[18] Manchester, R. N., Hobbs, G.B., Teoh, A. \& Hobbs, M., AJ, 2005, 129, 1993-2006 
[19] Marchetti, T., Rossi, E.M. and Brown, A.G.A. 2018, 1804, arXiv:1804.10607v1

[20] Marchetti, T., Rossi, E. M., \& Brown, A. G. A. 2018, ArXiv e-prints, arXiv:1804.10607

[21] Miyamoto \& Nagai, 1975, PASJ, 27, 533

[22] Navarro, Frenk \& White, 1996, ApJ, 462, 563

[23] Perets, H. B., \& Šubr, L., 2012, ApJ, 751, 133

[24] Poveda A., Ruiz J., Allen C., 1967, BOTT, 4, 86

[25] Shen, K. J., Boubert, D., Gänsicke, B. T., et al. 2018, ArXiv e-prints, arXiv:1804.11163

[26] Tauris, T.M., 2015, MNRAS Lett., 448, L6 